\title{
Resistencia a la corrosión a alta temperatura de recubrimientos NiCrAlY y NiCrFeNbMoTiAl depositados por APS
}

\author{
Corrosion Resistance at High Temperature of NiCrAlY and \\ NiCrFeNbMoTiAl Coatings Deposited by APS
}

Resistência à corrosão a alta temperatura de recobrimentos NiCrAlY e NiCrFeNbMoTiAl depositados por APS

Fecha de Recepción: 6 de septiembre de 2014

José Luis Tristancho-Reyes*

Fecha de Aceptación: 16 de octubre de 2014

José Guadalupe Chacón-Nava**

Facundo Almeraya-Calderón***

\section{Resumen}

La corrosión a alta temperatura de las tuberías utilizadas en equipos generadores de vapor (calderas) ha sido reconocida como un grave problema que trae consigo el adelgazamiento de éstas y, por consiguiente, la falla de los equipos. En la última década se han incrementado las investigaciones que involucran recubrimientos protectores que ayudan de alguna manera a prolongar la vida útil de estos equipos. Esta investigación determinó el comportamiento de los recubrimientos NiCrAlY y NiCrFeNbMoTiAl depositados por proyección térmica asistida por plasma (APS) sobre la aleación SA213 - T22 $\left(2 \frac{1}{4} \mathrm{Cr}-1 \mathrm{Mo}\right)$, en un ambiente corrosivo de $80 \% \mathrm{~V}_{2} \mathrm{O}_{5}$ $-20 \% \mathrm{~K}_{2} \mathrm{SO}_{4}$ a $800^{\circ} \mathrm{C}$. Los valores de la cinética de corrosión fueron determinados mediante resistencia a la polarización lineal (RPL) y espectroscopia de impedancia electroquímica (EIE). Los resultados obtenidos muestran una menor cinética de corrosión en el recubrimiento $\mathrm{NiCrFeNbMoTiAl} \mathrm{que} \mathrm{la} \mathrm{presentada} \mathrm{por} \mathrm{el} \mathrm{recubrimiento}$ NiCrAlY, corroborado por Microscopia Electrónica de Barrido (MEB).

Palabras clave: Corrosión a alta temperatura, Electroquímica, Recubrimientos, APS.

* Ph.D. Universidad Tecnológica de Pereira (Pereira-Risaralda, Colombia). josetris@utp.edu.co

** Ph.D. Centro de Investigación en Materiales Avanzados-CIMAV-(Chihuahua, México). jose.chacon@cimav.edu.mx

*** Ph. D. Autónoma de Nuevo León (Nuevo León, México). falmeraya.uanl.ciia@gmail.com 


\section{Abstract}

The corrosion at high temperature in pipes used in steam generating equipment (boilers), has been recognized as a serious problem, that results in the pipes thinning and therefore the equipments failure. In the last decade there has been a rise in investigations involving protective coatings to help, in some way to extend the useful life of these equipments. This investigation determined the NiCrFeNbMoTiAl and NiCrAlY coatings behavior, deposited by plasma-assisted thermal projection spraying (APS) on the alloy SA213 T22 $\left(2 \frac{1}{4} \mathrm{Cr}-1 \mathrm{Mo}\right)$, in acorrosive environment of $80 \% \mathrm{~V}_{2} \mathrm{O}_{5}-20 \% \mathrm{~K}_{2} \mathrm{SO}_{4}$ to $800{ }^{\circ} \mathrm{C}$. The kinetics corrosion values were determined by linear polarization resistance (LPR) and electrochemical impedance spectroscopy (EIS). The results obtained show a lower kinetic corrosion on the NiCrFeNbMoTiAl coating than the NiCrAlY coating, confirmed by the SEM, Scanning Electron Microscopy.

Keywords: Hot Corrosion, Electrochemical, Coatings, APS.

\section{Resumo}

A corrosão a alta temperatura dos encanamentos utilizados em equipamentos geradores de vapor (caldeiras) tem sido reconhecida como um grave problema que traz o adelgaçamento destas e, por conseguinte, a falha dos equipamentos. Na última década tem se incrementado as pesquisas que envolvem recobrimentos protetores que ajudam de alguma maneira a prolongar a vida útil destes equipamentos. Esta pesquisa determinou o comportamento dos recobrimentos NiCrAlY e NiCrFeNbMoTiAl depositados por projeção térmica assistida por plasma (APS)

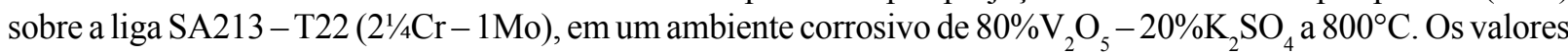
da cinética de corrosão foram determinados mediante resistência à polarização linear (RPL) e espectroscopia de impedância eletroquímica (EIE). Os resultados obtidos mostram uma menor cinética de corrosão no recobrimento $\mathrm{NiCrFeNbMoTiAl} \mathrm{que} \mathrm{a} \mathrm{apresentada} \mathrm{pelo} \mathrm{recobrimento} \mathrm{NiCrAlY,} \mathrm{corroborado} \mathrm{por} \mathrm{Microscopia} \mathrm{Eletrônica} \mathrm{de}$ Varredura (MEV).

Palavras chave: Corrosão a alta temperatura, Eletroquímica, Recobrimentos, APS. 


\section{INTRODUCCIÓN}

La corrosión a alta temperatura por sales fundidas ha sido considerada uno de los mayores problemas que se presentan en la industria generadora de energía eléctrica, especialmente en los componentes de equipos generadores de vapor (calderas) que operan a elevadas temperaturas $\left(600-1100^{\circ} \mathrm{C}\right)$, en donde se presenta el contacto directo de los materiales metálicos con los gases producto de la combustión que contienen impurezas inorgánicas. Dependiendo de las condiciones de diseño de los bancos intercambiadores de calor (sobrecalentadores y recalentadores), en algunas regiones específicas se forman finas películas de sales fundidas sobre las superficies metálicas, dando origen a una condición altamente corrosiva.

El uso de recubrimientos metálicos se ha extendido cada vez más a diversos sectores industriales, donde se requiere resistencia a elevada temperatura, a los ambientes altamente agresivos y al desgaste. Desde los años 70 [1,2], los recubrimientos metálicos, que actúan como barreras térmicas, han incrementado la temperatura de trabajo en el interior de los equipos generadores de vapor (calderas) y, a su vez, han disminuido la temperatura de los materiales metálicos que los conforman, incrementando su vida útil y reduciendo el consumo de combustible para generar la misma energía [3].

Un recubrimiento para uso a altas temperaturas puede ser definido como una capa superficial de un material que puede ser metálico, cerámico o de una combinación de estos, que puede evitar o inhibir la interacción directa entre la aleación base y un ambiente potencialmente dañino. El daño causado por esta interacción puede traducirse en pérdida de substrato por oxidación o corrosión a alta temperatura o por la pérdida de la resistencia mecánica del sustrato debido a la difusión de especies perjudiciales a la aleación base a altas temperaturas [4].

Los recubrimientos metálicos empleados no suelen ser barreras inertes, sino, mas bien, compuestos cuyos elementos reaccionan con el oxígeno del ambiente para formar capas de óxidos densas u adherentes que inhiben la difusión de especies dañinas, tales como más oxígeno, nitrógeno, azufre, vanadio, sodio y potasio, hacia el sustrato metálico; los recubrimientos deberán, en consecuencia, ser ricos en elementos tales como aluminio, níquel, cromo y silicio, entre otros, que sean capaces de formar estos óxidos protectores. Esencialmente, los recubrimientos empleados a altas temperaturas pueden considerarse reservas de estos elementos, que, en la medida en que se presenten desprendimientos causados por ciclos térmicos o daños mecánicos, puedan regenerar más capa de óxido protector. Así, dada la naturaleza del mecanismo de protección que ofrecen, la vida útil de los recubrimientos protectores depende de su habilidad para formar las capas de óxido protector y para retenerlas y reemplazarlas si así se requiere.

En general, para que un recubrimiento represente beneficios tanto económicos como técnicos debe cumplir con una serie de requisitos, entre otros:

Debe proporcionar un incremento de vida útil de los componentes metálicos a la temperatura y en el ambiente de servicio, siendo resistente a la oxidación y a la corrosión a alta temperatura, así como estable frente a la interdifusión substratorecubrimiento.

$>$ El recubrimiento no debe afectar significativamente las propiedades mecánicas del componente, en lo que respecta a la resistencia a la fluencia, a la fatiga y a los choques térmicos.

Los materiales del recubrimiento y el proceso de su aplicación deben ser aceptables no solo económicamente, sino también medioambientalmente, y no deben implicar una proporción significativa de elementos considerados "estratégicos".

$>$ El proceso de aplicación debe ser compatible con el tamaño y la geometría de los componentes.

El método de Proyección Térmica Asistida por Plasma (APS, por su sigla en inglés) es una técnica potente $\mathrm{y}$ ampliamente usada para producir recubrimientos protectores sobre una gran cantidad de sustratos. La formación de estos recubrimientos se basa en los sucesivos impactos de partículas fundidas sobre la superficie del sustrato, o sobre partículas ya depositadas, obteniéndose una estructura laminar característica [5]. 
La Espectroscopia de Impedancia Electroquímica (EIE) es una técnica de análisis no perturbativo, rápida y reproducible. Mediante EIE se determina la impedancia del sistema como respuesta a una señal de corriente alterna que varía en un amplio intervalo de frecuencia; estas respuestas pueden caracterizarse por medio de circuitos eléctricos equivalentes que usan combinaciones en serie o en paralelo de resistencias (R) y capacitancias (C) que representan el proceso que se está llevando a cabo [6].

En esta investigación se evalúo la resistencia a la corrosión a alta temperatura de los recubrimientos NiCrAlY y NiCrFeNbTiAl depositados mediante APS sobre la aleación SA213-T22 (21/4Cr-1Mo), en un ambiente corrosivo de $80 \% \mathrm{~V}_{2} \mathrm{O}_{5}-20 \% \mathrm{~K}_{2} \mathrm{SO}_{4}$ a $800^{\circ} \mathrm{C}$, mediante las técnicas electroquímicas de Resistencia a la Polarización Lineal (RPL) y EIE.

\section{Desarkollo experimental}

A fin de evaluar la resistencia a la corrosión a alta temperatura por sales fundidas en los recubrimientos NiCrAlY y NiCrFeNbMoTiAl, se maquinaron probetas cilíndricas de $5 \mathrm{~mm}$ de diámetro y $25 \mathrm{~mm}$ de altura de la aleación SA213-T22 (21/4Cr-1Mo), que es de uso común en centrales termoeléctricas y cuya composición se muestra en la Tabla 1. Los recubrimientos protectores fueron depositados mediante la técnica de APS, bajo las siguientes condiciones de aplicación:

$>$ Presión gas primario (Ar): $50 \mathrm{psi}$

$>$ Presión gas secundario (He): 110 psi

$>$ Presión gas de arrastre (Ar): 35 psi

$>$ Corriente: $500 \mathrm{~A}$

$>$ Voltaje: $35.8 \mathrm{~V}$

TABLA 1

Composición QuímiCA DE LA ALEACIÓN SAE -213 (\% EN PESO)

\begin{tabular}{cccccccc}
\hline $\mathrm{Cr}$ & $\mathrm{C}$ & $\mathrm{Mn}$ & $\mathrm{Mo}$ & $\mathrm{P}$ & $\mathrm{S}$ & $\mathrm{Si}$ & $\mathrm{Fe}$ \\
\hline 2.18 & 0.09 & 0.48 & 0.93 & 0.96 & 0.01 & 0.30 & Balance. \\
\hline
\end{tabular}

Para realizar los ensayos electroquímicos se hizo uso de un arreglo de tres electrodos: un electrodo de trabajo, aleación SA213-T22 recubierta con NiCrAlY y $\mathrm{NiCrFeNbMoTiAl,} \mathrm{y} \mathrm{como} \mathrm{electrodos} \mathrm{de} \mathrm{referencia}$ y contraelectrodo se utilizó alambre de platino de alta pureza, de $1 \mathrm{~mm}$ de diámetro, embebidos en tubos de cuarzo sellados por los extremos con cemento cerámico. El arreglo fue introducido en horno para ensayos de corrosión a alta temperatura (Fig. 1).

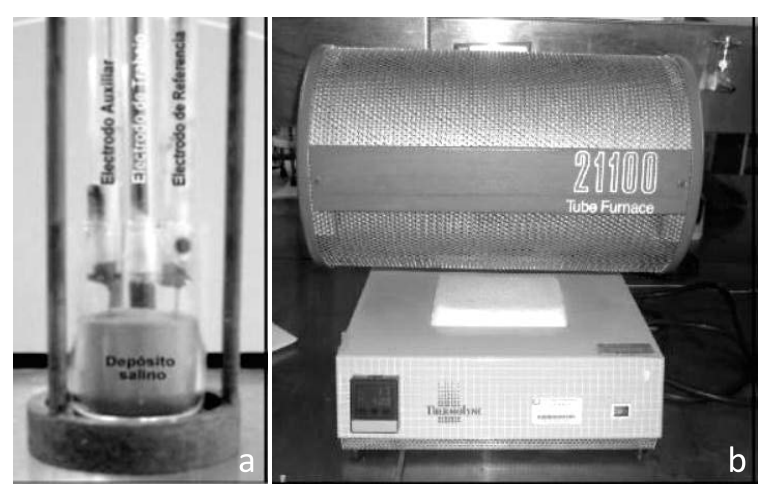

Fig. 1. (a) Celda electroquímica, (b) Horno para ensayos de corrosión a alta temperatura

El agente corrosivo utilizado consistió en una mezcla de $80 \%$ de $\mathrm{V}_{2} \mathrm{O}_{5}$ (pentóxido de vanadio) y $20 \%$ de $\mathrm{K}_{2} \mathrm{SO}_{4}$ (sulfato de potasio) en peso, grado analítico, garantizando su alta pureza. Esta mezcla presenta su punto de fusión a una temperatura de $620^{\circ} \mathrm{C}$, y se asemeja a las cenizas producto de la combustión en las calderas de las centrales termoeléctricas. Antes de cada ensayo la mezcla fue sometida a agitación mecánica durante un tiempo de 20 minutos, homogeneizándola completamente. Para cada ensayo se utilizaron $30 \mathrm{~g}$ de la mezcla salina.

En el momento de introducir la celda electroquímica en la cámara del horno, éste se encontraba a temperatura ambiente, se encendió y se llevó a temperatura de trabajo, $800^{\circ} \mathrm{C}$; una vez alcanzada esta temperatura se mantuvo durante aproximadamente $15 \mathrm{~min}$. a fin de lograr la estabilización del sistema. A partir de este momento se aplicaron las técnicas electroquímicas de RPL y EIE, obteniéndose la cinética de corrosión de los dos sistemas de encubrimientos. La duración del ensayo, a partir del momento de estabilización del sistema, fue de 1 hora 20 minutos, aproximadamente. 
Para la técnica RPL se realizó un barrido de $20 \mathrm{mV}$ respecto al potencial de equilibrio a una velocidad de $10 \mathrm{mV} / \mathrm{min}$.; esta técnica, al requerir solo la aplicación de pequeñas polarizaciones, no altera la superficie del electrodo de trabajo [7]. Para la técnica de EIE se realizó un barrido de frecuencia desde $0,01 \mathrm{mHz}$ hasta $10 \mathrm{KHz}$; al atravesar la corriente alterna, la interface del electrodo de trabajo se divide en dos: una corriente motivada por el comportamiento capacitivo de la interface, y la otra como una corriente de transferencia que se refiere al intercambio de cargas de las reacciones electroquímicas [8].

\section{RESULTADOS Y ANÁLISIS}

En las Figuras 2(a) y 2(b) se muestran las micrografías MEB de las interfaces metal baserecubrimiento NiCrAlY y metal base-recubrimiento NiCrFeNbMoTiAl, respectivamente, observándose capas de recubrimientos poco porosas, homogéneas y con buena adherencia que actúan como barreras protectoras que impiden el contacto directo del metal base con el agente corrosivo.

En las Figuras 3(a) y 3(b) se muestran los diagramas de resistencia a la polarización, y en la Tabla 2 se presentan los valores de resistencia a la polarización (Rp) y los valores de velocidad de corrosión (Vcorr) obtenidos al exponer los recubrimientos NiCrAlY y $\mathrm{NiCrFeNbMoTiAl} \mathrm{al} \mathrm{ataque} \mathrm{corrosivo} \mathrm{por} \mathrm{sales}$ fundidas $80 \% \mathrm{~V} 2 \mathrm{O} 5-20 \% \mathrm{~K} 2 \mathrm{SO} 4$ a $800^{\circ} \mathrm{C}$.
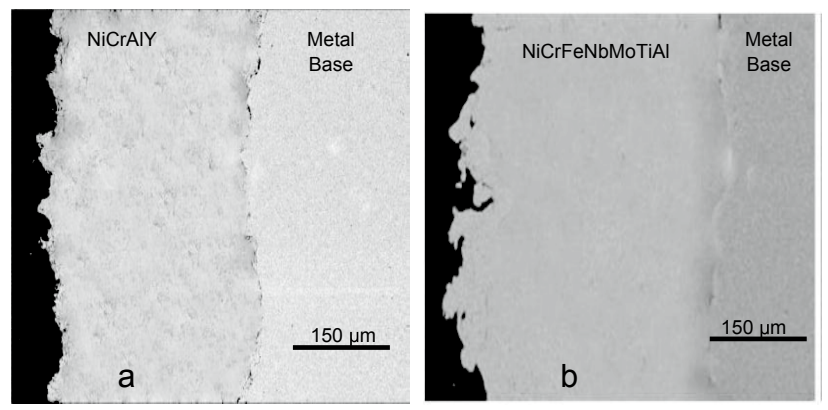

Fig. 2. (a) Interface Metal Base-Recubrimiento NiCrAlY; (b) Interface Metal Base-Recubrimiento $\mathrm{NiCrFeNbMoTiAl.}$
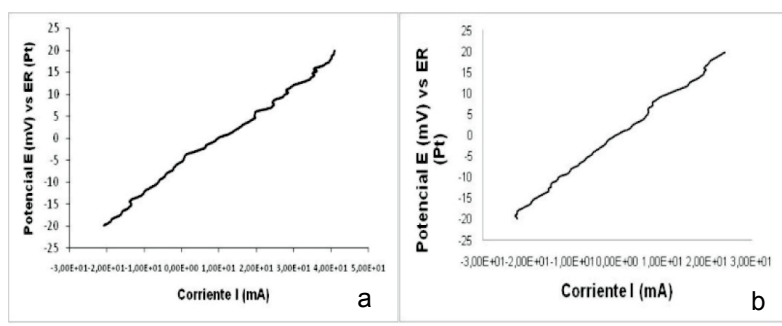

Fig. 3. Diagramas de Resistencia a la Polarización (Rp): (a) Recubrimiento NiCrAlY y (b) Recubrimiento $\mathrm{NiCrFeNbMoTiAl.}$

\section{TABLA 2}

VALORES DE RESISTENCIA A LA POLARIZACIÓN LINEAL Y VELOCIDADES DE CORROSIÓN

\begin{tabular}{ccc}
\hline Recubrimiento & $\operatorname{Rp}(\Omega)$ & $\begin{array}{c}\text { Vcorr } \\
\left(\mathrm{mA} / \mathrm{cm}^{2}\right)\end{array}$ \\
\hline NiCrAlY & 0.8191 & 0.1715 \\
\hline NiCrFeNbMoTiAl & 0.7505 & 0.2391 \\
\hline
\end{tabular}

Para determinar la cinética de corrosión se hizo uso de la ecuación de Stern and Geary (ecuación 1), que relaciona la corriente de corrosión Icorr $\left(\mathrm{mA} / \mathrm{cm}^{2}\right)$ con la resistencia a la polarización $\operatorname{Rp}(\Omega)$.

$$
R_{p}=\beta / I_{\text {corr }}
$$

Siendo $\beta$ una constante adimensional dada en función de las pendientes anódica y catódica determinadas de las curvas de polarización de cada ensayo (ecuación 2).

$$
\beta=\frac{\left(b_{a} * b_{c}\right)}{2.303\left(b_{a}+b_{c}\right)}
$$

La velocidad de corrosión estará dada en función de la densidad de corriente de corrosión, del peso específico y de la densidad de cada recubrimiento de la siguiente forma (ecuación 3):

$$
V_{\text {corr }}=3.27 * 10^{-3}\left(I_{\text {corr }} *\left(\frac{P . E}{\rho}\right)\right)
$$

De los valores obtenidos por la técnica de RPL se observa un mayor valor de resistencia a la polarización (Rp) al someter el recubrimiento NiCrAlY en un agente corrosivo $80 \% \mathrm{~V} 2 \mathrm{O} 5-20 \% \mathrm{~K} 2 \mathrm{SO} 4$ a $800^{\circ} \mathrm{C}$, siendo éste más resistente al ataque corrosivo que el recubrimiento $\mathrm{NiCrFeNbMoTiAl}$, que muestra mayor cinética de corrosión. 
En la Tabla 3 se muestran los valores de resistencia a la transferencia de carga (Rtc) y los valores de velocidad de corrosión (Vcorr) determinados por espectroscopia de impedancia electroquímica (EIE). Los diagramas de Nyquist obtenidos para cada ensayo se muestran en las Figuras 4(a) y 4(b).

En los diagramas de Nyquist se observa la presencia de dos semicircunferencias: una a bajas frecuencias, que puede ser atribuida al comportamiento de la capa de recubrimiento metálico (representada por RR // CR), $\mathrm{y}$ otra a altas frecuencias, que describe el fenómeno de corrosión (representado por Rtc // Cdl).

Debido a que el proceso corrosivo es controlado por la transferencia de carga a través del recubrimiento y no por la difusión de especies en el depósito de sales fundidas, el radio de la segunda semicircunferencia debe ser mayor que el de la primera, y el valor total de la impedancia electroquímica estará dado por (4) [9].

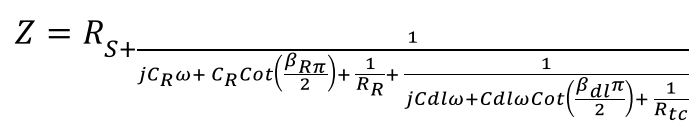

Donde $\mathrm{R}$ y $\mathrm{dl}$ corresponden a los coeficientes de dispersión en el primer y segundo ciclo de la capacitancia; en consecuencia, $\mathrm{CRCot}(\mathrm{R} / 2)$ y $\mathrm{CdlCot}(\mathrm{dl} / 2)$ son elementos de impedancia causados por el efecto de dispersión.

Al presentarse una capa de recubrimiento metálico protector sobre la superficie metálica, la cinética del proceso corrosivo puede disminuirse; este fenómeno puede ser representado por el circuito equivalente mostrado en las Figuras 5(a) y 5(b) para los recubrimientos NiCrAlY y NiCrFeNbMoTiAl, respectivamente [10].

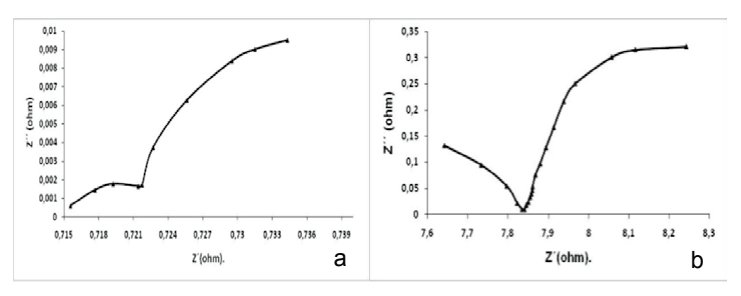

Fig. 4. Diagramas de Nyquist: (a) Recubrimiento NiCrAlY y (b) Recubrimiento NiCrFeNbMoTiAl
Donde: RS es la resistencia de la solución (también conocida como la resistencia no compensada), y es asociada con el electrolito (sales fundidas) y se asume invariante durante la aplicación de la técnica AC, independiente de la geometría de la muestra y del tiempo de duración del ensayo [11 - 13].

$\mathrm{RR}$ es la resistencia de la capa de recubrimiento (resistencia de poro o resistencia a la transferencia de iones en la capa de recubrimiento); CR corresponde a la capacitancia de la capa de recubrimiento; Rtc es la resistencia a la transferencia de carga (resistencia interfacial o resistencia a la corrosión) y Cdl corresponde a la capacitancia de la doble capa; estos dos últimos son asociados al sustrato metálico o, posiblemente, a la interface sustrato metálico/recubrimiento $[11,12,13]$.

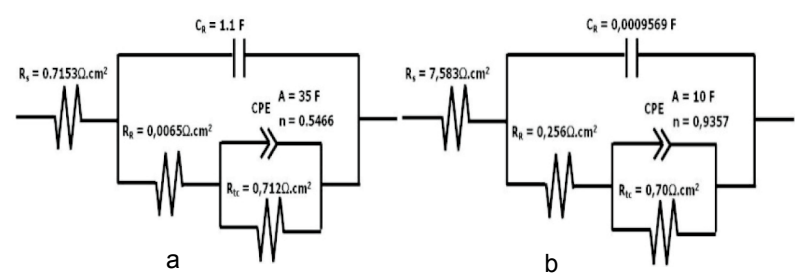

Fig. 5. Circuitos equivalentes: (a) Recubrimiento NiCrAlY y (b) Recubrimiento NiCrFeNbMoTiAl

De los valores obtenidos a partir de EIE, se aprecia mayor cinética de corrosión al exponer el recubrimiento $\mathrm{NiCrFeNbMoTiAl} \mathrm{al} \mathrm{ataque} \mathrm{corrosivo}$ por sales fundidas $80 \% \mathrm{~V} 2 \mathrm{O} 5-20 \% \mathrm{~K} 2 \mathrm{SO} 4$ a $800^{\circ} \mathrm{C}$ que el valor obtenido al someter el recubrimiento NiCrAlY al mismo ambiente corrosivo.

El análisis por MEB corrobora que la mayor severidad de ataque se presentó en el recubrimiento NiCrFeNbMoTiAl, y que el recubrimiento NiCrAlY tuvo mejor comportamiento al ser expuesto a ataque corrosivo por sales fundidas.

\section{Conclusiones}

El recubrimiento NiCrAlY presentó mejor comportamiento ante el ataque corrosivo a alta temperatura por sales fundidas, al obtenerse mayores valores de resistencia a la polarización (Rp) y resistencia a la transferencia de carga (Rtc); los valores de velocidad de corrosión determinados para este recubrimiento fueron 
menores a los presentados por el recubrimiento NiCrFeNbMoTiAl.

Mediante MEB se corroboró la mayor severidad de ataque del agente corrosivo sobre el recubrimiento $\mathrm{NiCrFeNbMoTiAl}$, expresado en mayor deterioro, es decir, mayor disminución del espesor de la capa de éste respecto al observado en la capa del recubrimiento NiCrAlY.

\section{Agradecimientos}

Los autores expresan sus más sinceros agradecimientos a Colciencias, Conacit, Cimav y a la Universidad Tecnológica de Pereira, por el apoyo económico para la realización de esta investigación.

\section{REFERENCIAS}

[1] M. Pomeroy, "Coatings for gas turbine materials and long term stability issues", Materials and Desing, vol. 26, pp. 223-231, 2005.

[2] J. Gómez, A. Salazar, C. Múnez, V. Utrilla y P. Poza, "Análisis de la degradación de recubrimientos de barrera térmica por espectroscopia de impedancia electroquímica", Boletín de la Sociedad Española de Cerámica y Vidrio, vol. 46, pp. 232-239, 2007.

[3] A. Agüero, "Recubrimientos contra la corrosión a alta temperatura, para componentes de turbinas de gas", Revista de Metalurgia, vol. 43, pp. 384398, 2007.

[4] B. Sidhu and S. Prakash, "Evaluation of the corrosion behavior of plasma - sprayed Ni3Al coatings on steel in oxidation and molten salts environments at $900^{\circ} \mathrm{C}$ ". Surface and Coatings Technology, vol. 166, pp. 89-100, 2003.

[5] B. Hinderliter, S. Croll, D. Tallman, Q. Su and G. Bierwagen, "Interpretation of EIS data from accelerated exposure of coated metals based on modeling of coatings physical properties", Electrochemical ACTA, vol. 51, pp. 4505-4515, 2006.
[6] F. Floyd, S. Avudalappan, J. Gibson, B. Mehta, P. Smith, T. Povder and J. Escarsega, "Using electrochemical impedance spectroscopy to predict the corrosion resistance of unexposed coated metal panels", Progress in Organic Coatings, vol. 50, pp. 8-34, 2009.

[7] ASTM G-102. Calculation of corrosion rates and related information from electrochemical measurements. American Society for Testing and Materials.EE.UU.,1997.

[8] ASTM G-59. Standard test method for conducting potentiodynamic polarization resistance measurements.American Society for Testing and Materials. EE.UU.,1997.

[9] ASTM G-3. Standard test for conventions applicable to electrochemical measurements in corrosion testing.American Society for Testing and Materials. EE.UU.,1999.

[10] C. Zhang, C. Zhou, S. Gong, H. Li and H. $\mathrm{Xu}$, "Evaluation of thermal barrier coating exposed to different oxygen partial pressure environments by impedance spectroscopy", Surface and Coatings Technology, vol. 201, pp. 446-451, 2006.

[11] J. Tristancho, J. Chacón, D. Peña, C. Gaona, J. Gonzáles, A. Martínez and F. Almeraya, "Hot corrosiónbehaviour of $\mathrm{NiCrFeNbMoTiAl}$ coating in molten salts at $700^{\circ} \mathrm{C}$ by electrochemical techniques", International Journal of Electrochemical Science, vol. 6, pp. 4323-441, 2011.

[12] C. Zeng, W. Wang and W. Wu, "Electrochemical Impedance models for molten salts corrosion", Corrosion Science, vol. 43, pp. 787-801, 2001.

[13] J. Tristancho, M. Sánchez, R. Sandoval, V. Orozco, F. Almeraya, J. Chacón, J. Gonzáles and A. Martínez, "Electrochemical impedance spectroscopy investigation of alloy inconel 718 in molten salts at high temperatura", International Journal of Electrchemical Science, vol. 6, pp. 419-431, 2001. 